\title{
Ancylostomiasis due to Ancylostoma Ceylanicum
}

National Cancer Institute

\section{Source}

National Cancer Institute. Ancylostomiasis due to Ancylostoma Ceylanicum. NCI

Thesaurus. Code C35366.

Infection by Ancylostoma ceylanicum. 